\title{
El cambio climático y el desarrollo
}

\author{
Jorge Álvarez Lam \\ Consultor del PNUD \\ Ingeniería Industrial n. 28, 2010, ISSN 1025-9929, pp 25-40 \\ Recibido: 3 de junio del 2010 / Aprobado: 13 de julio del 2010
}

\begin{abstract}
RESUMEN: El cambio climático nos enfrenta a nuevos retos y desafíos que ponen en riesgo nuestro futuro desarrollo; por otra parte, el cambio climático puede convertirse en una oportunidad que nos fuerce a ser más eficientes en el uso de los recursos y con ello más competitivos a escala internacional. El país que esté preparado para enfrentar estos retos podrá evitar la pérdida de recursos internos significativos y aprovechar los flujos financieros que se generen en el marco de las negociaciones mundiales sobre cambio climático.
\end{abstract}

Palabras clave: cambio climático / desarrollo sostenible

\section{Climate change and development}

ABSTRACT: Climate change confronts us with new challenges that threaten our future development on the other hand climate change may become an opportunity to force us to be more efficient in the use of our resources and thus more competitive at international level. The country that is prepared to face these challenges can avoid the loss of significant internal resources and utilize the financial flows generated in the context of global negotiations on climate change.

Keywords: climate change / sustainable development 


\section{ALGUNOS CONCEPTOS}

El cambio climático es uno de los principales problemas ambientales que viene afectando la economía peruana y es el problema más grande que va a enfrentar la humanidad en los próximos años. Por ello, el sistema climático global, tal cual lo conocemos, será modificado, afectando los ecosistemas y algunos recursos naturales que son base para nuestro desarrollo y sustento.

Pero ¿cómo se generó este cambio climático? Para entender esto, repacemos algunos conceptos con apoyo del siguiente gráfico:

\section{Gráfico 1}

El cambio climático y el efecto invernadero

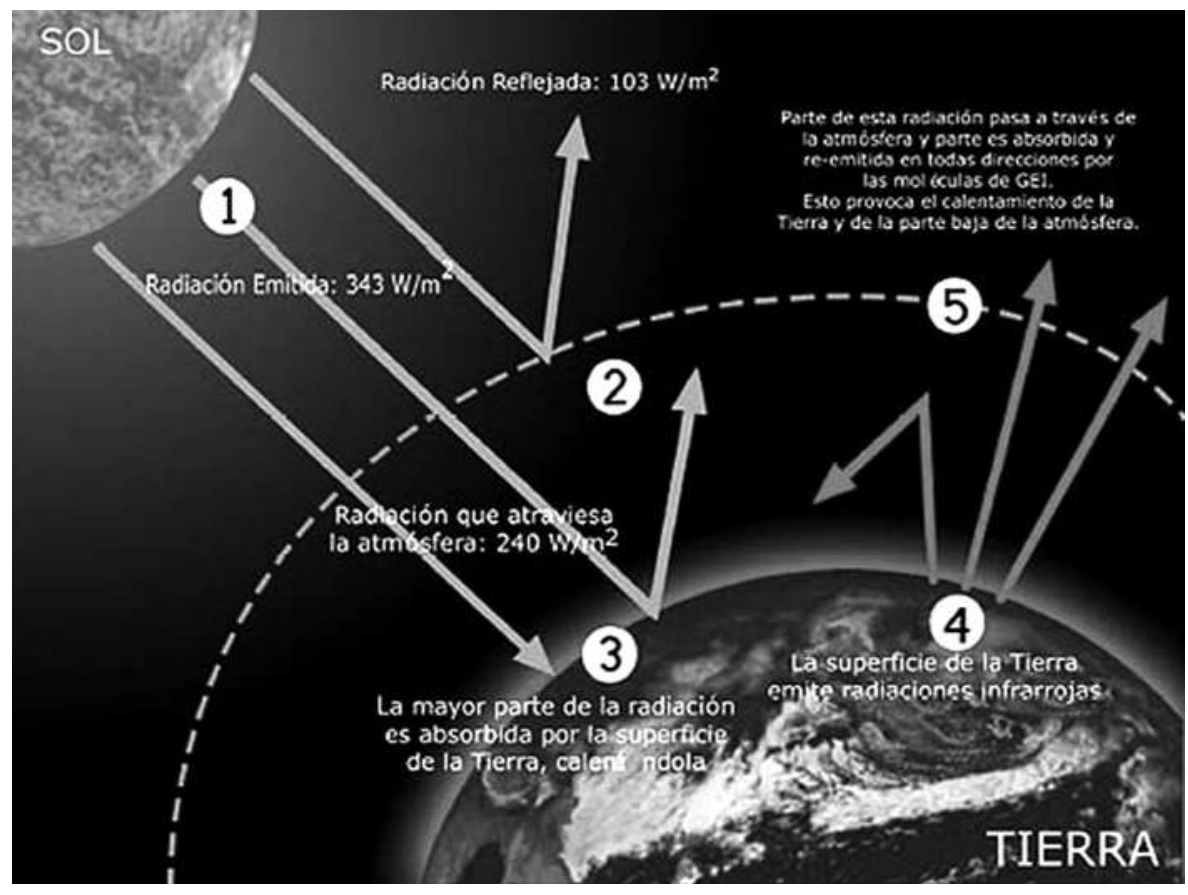

Fuente: IPCC. 
El efecto invernadero es un fenómeno natural que siempre ha existido y permite la vida en el planeta; este efecto hace posible almacenar calor en la superficie de la Tierra gracias a la presencia de unos gases denominados de efecto invernadero (GEI). Si no hubiera estos gases en la atmósfera, la temperatura en las noches descendería por debajo de los $10^{\circ} \mathrm{C}$, como en la Luna, que haría que la vida tal como la conocemos no exista en nuestro planeta. Sin embargo, este fenómeno, que es natural y beneficioso para la existencia del hombre, se convirtió en un problema debido a que el ser humano, en su afán por el desarrollo (desde la era industrial hasta la actualidad), comenzó a emitir demasiados gases de efecto invernadero, haciendo que la capacidad de almacenar calor se incremente, dando origen a un calentamiento general del planeta, denominado calentamiento global, que ha generado como consecuencia la modificación del sistema climático mundial, distorsionando los ciclos climáticos del planeta, fenómeno que se conoce como cambio climático. A veces es difícil entender qué es el calentamiento global, cuando en nuestras zonas altoandinas las heladas son cada vez más frecuentes y severas. Lo que sucede es que al modificarse el sistema climático mundial (cambio climático) unas zonas serán más frías y otras más calientes, pero en general habrá un incremento de la temperatura promedio del planeta (calentamiento global).

Es cierto que existen muchos detractores de la denominación cambio climático, quienes indican que hace muchos años las temperaturas y concentraciones de gases de efecto invernadero alcanzaron valores superiores a los actuales, sin embargo estas variaciones se han dado en periodos muy largos, que han permitido la adaptación de algunas especies y la desaparición de otras. Pero en esta ocasión, la temperatura se ha ido modificando en los últimos 200 años, tiempo que no permite que muchas especies se adapten o al menos no de manera natural, afectando su existencia, así como la de las especies que viven de ellas, incluido el ser humano. 


\section{Gráfico 2 \\ Incremento de las concentraciones de GEI a escala global}

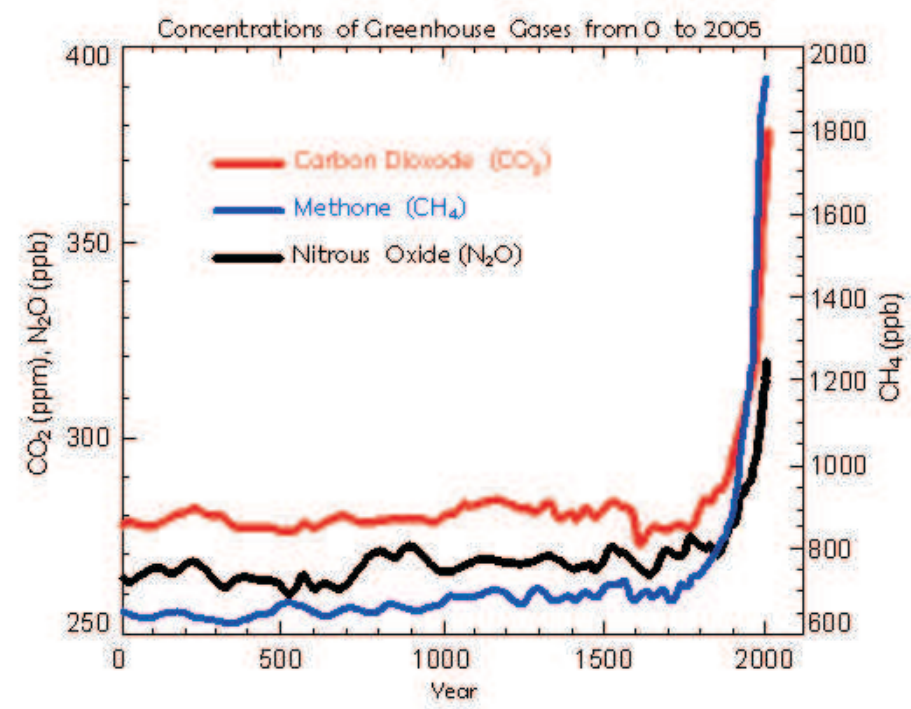

Fuente: IPCC 2007.

Como se ve en gráfico N.$^{\circ} 2$ :

- Aumento desde 1750 debido a la actividad industrial.

- Una subida rápida durante los últimos 50 años.

- La concentración atmosférica de CO2 se había mantenido por debajo de 300 ppm durante los últimos 600.000 años.

- Actualmente se encuentra en 380 ppm y con tendencia a subir.

Uno de los mayores problemas radica en que, aun hoy, la mayoría de personas considera el cambio climático como un asunto ambiental, cuando en realidad se trata de un tema de sostenibilidad económica de los países (desarrollo económico sustentable), ya que si estos quieren seguir desarrollándose, tendrán que ser más conscientes y eficientes en su manera de desarrollarse, y además adaptar este desarrollo a las condiciones climáticas futuras. 
El cambio climático puede ser un freno al desarrollo económico, por ello, la inclusión de los escenarios climáticos en la planificación será una clave para garantizar este desarrollo.

\section{NUESTRO PROBLEMA}

Los efectos adversos del cambio climático ya son evidentes en muchas zonas del mundo y las consecuencias son cada vez más claras; sin embargo, para países megadiversos como el Perú, donde tenemos 28 de los 35 climas y 84 de las 114 zonas de vida existentes en el planeta, nos es difícil predecir cuál será el comportamiento del clima en cada una de estas zonas en el futuro, y sumadas a esto las condiciones de pobreza y pobreza extrema de algunas de nuestras localidades, nos sitúan como un país altamente vulnerable al cambio climático, al no estar aún preparados para responder a estos eventos.

No es que en el Perú no se haya avanzado en la investigación sobre el cambio climático, ya que nuestro país es considerado un referente en cuanto al tema en la región, debido entre otras cosas a la capacidad del Senamhi para generar escenarios climáticos futuros en las cuencas hidrográficas ${ }^{1}$ o también por las metodologías empleadas por el Ministerio del Ambiente (Minam) para establecer y priorizar zonas vulnerables $^{2}$ o plantear medidas de adaptación sobre la base de los escenarios desarrollados; pero ocurre que estos estudios requieren de tiempo y recursos económicos con los que no se cuenta.

También debemos considerar que por la megadiversidad del Perú se necesita más detalle en estos análisis, ya que es preciso que las variaciones climáticas se den de acuerdo con las propias condiciones de cada cuenca o subcuenca. Asimismo, cada propuesta de adaptación será diferente según las características de cada localidad, y en esta propuesta, los aspectos sociales también deben ser tomados en cuenta si se quiere sea aceptada por la población en un corto plazo, pues no se puede atentar contra sus propias costumbres. De allí que el tema

1 A la fecha se han desarrollado escenarios climáticos nacionales y para seis cuencas: Santa Teresa, Shulcas, Mantaro, Chira-Piura, Santa, Altomayo.

2 El Perú cuenta con un mapa nacional de vulnerabilidad del cambio climático que se va perfeccionando a medida que avanza el conocimiento del tema (Minam. www.minam. gob.pe). 
del cambio climático y las medidas que se tomen no pueden decidirse como si se tratara de un catálogo de cuestiones por ejecutar. Hay que analizar la situación cuenca por cuenca y comunidad por comunidad, pues la mejor medida de adaptación para una puede no serlo para otras.

Sin embargo, los estudios nacionales desarrollados ya pueden dar indicios de lo que sucederá en el futuro, y considero que no es necesario esperar que los estudios estén terminados para tomar cartas en el asunto, ya que se pueden ir adoptando medidas mientras avanza la investigación. A continuación se resumen los resultados más resaltantes de los estudios nacionales de caracterización climática (cómo se ha comportado el clima en el pasado) y de los escenarios climáticos futuros:

\section{- Caracterización climática nacional}

- Las temperaturas máximas y mínimas se han incrementado hasta en $0,2^{\circ} \mathrm{C} /$ década en casi todo el territorio.

- Los periodos secos se han incrementado en mayor intensidad que los periodos húmedos a escala nacional.

- La intensidad de las precipitaciones está incrementándose en la costa y sierra norte, mientras que está disminuyendo en la sierra central, la sierra sur presenta variaciones moderadas en ambos sentidos.

\section{- Escenarios climáticos nacionales}

- Las lluvias extremas estimadas muestran un probable decrecimiento en los próximos 30 años en gran parte del territorio.

- En general se muestra un incremento de la temperatura máxima de hasta $1,6^{\circ} \mathrm{C}$ en promedio $\left(0,53^{\circ} \mathrm{C} /\right.$ década $)$, y de hasta $1,4^{\circ} \mathrm{C}$ para la temperatura mínima $\left(0,47^{\circ} \mathrm{C} /\right.$ década $)$ en los próximos 30 años.

- Los mayores incrementos se presentarían en la costa y sierra norte, luego en la selva norte y en la sierra central y sur del país.

Pero estos cambios, que a simple vista son ínfimos (incremento de $1,6^{\circ} \mathrm{C}$ en 30 años), ¿cómo pueden afectar nuestro desarrollo? Lo que hay que entender primero es que este dato es un promedio nacional y al mirar detalladamente esta información encontramos que en muchas zonas las variaciones serán mayores tanto hacia arriba como hacia abajo, pudiendo generar consecuencias diferenciadas según el lugar 
donde uno se encuentre. Por otro lado, no hay que analizar estos valores comparándolos con los que un ser humano puede o no soportar, ya que muchas especies que permiten la subsistencia del hombre no resisten ni medio grado centígrado de variación en la temperatura y ese es el problema fundamental para el Perú. Cuando el ser humano tiene frío se abriga y cuando tiene calor se desabriga, pero las plantas desaparecen, y los animales simplemente desaparecen o migran si pueden hacerlo; estas variaciones ponen en riesgo nuestro sustento, sin ir contra la pérdida de diversidad biológica, que es otro tema para analizar.

El Perú es considerado un país altamente vulnerable a los efectos adversos del cambio climático, porque presenta cuatro de las cinco características de vulnerabilidad reconocidas por la Convención Marco de las Naciones Unidas sobre Cambio Climático (Cmnucc) (tabla 1). Adicionalmente, el país continúa con su crecimiento poblacional y la ocupación económica del territorio, con persistencia de la pobreza, los ecosistemas amenazados, los glaciares tropicales en reducción, los problemas de distribución de recursos hídricos por su geografía diversa y mayormente agreste, y por la actividad económica altamente dependiente del clima.

Tabla 1

Perú, país vulnerable a los efectos adversos del cambio climático

\begin{tabular}{lc} 
Características reconocidas por la Cmnucc & Perú \\
\hline Países de baja altitud y países insulares & No \\
Países con zonas costeras bajas & Sí \\
$\begin{array}{l}\text { Zonas áridas y semiáridas; zonas con cobertura forestal y zonas expuestas a } \\
\text { deterioro forestal }\end{array}$ & Sí \\
Zonas expuestas a inundaciones, sequías y desertificación & Sí \\
Países con zonas propensas a los desastres naturales & Sí \\
$\begin{array}{l}\text { Países en desarrollo con ecosistemas montañosos frágiles; los países con zonas } \\
\text { de ecosistemas frágiles, incluidos los ecosistemas montañosos }\end{array}$ & Sí \\
Los países con zonas de alta contaminación atmosférica urbana & Sí \\
Los países cuyas economías dependen en gran medida de los ingresos generados & \\
por la producción, el procesamiento y la explotación de combustibles fósiles & \\
y productos asociados de energía intensiva o de su consumo & Sí \\
Países sin litoral y los países de tránsito & No \\
\hline
\end{tabular}

Fuente: Cmnucc 1992. 
Al analizar algunos de los impactos del cambio climático que ya desde el 2007 han sido alertados por el Panel Intergubernamental de Cambio Climático (IPCC por sus siglas en inglés), institución que agrupa a más de tres mil científicos del mundo (incluidos destacados científicos peruanos), se evidencia rápidamente cómo este fenómeno nos puede afectar si no estamos preparados para afrontar estos nuevos desafíos, y en algunos casos sacar provecho de este:

- La temperatura promedio podría subir entre 1,8 y 5, $8^{\circ} \mathrm{C}$.- Traerá consigo la modificación de los ciclos de cultivo y pérdidas de cosechas. Se deberán evaluar nuevas variedades resistentes a estos cambios si queremos garantizar la seguridad alimentaria en el Perú, además de evaluar las oportunidades que estas nuevas condiciones traerán en nuestras localidades, es probable que algunas especies que hoy son imposibles de cultivar en el futuro se pueda hacerlo. Se derretirán los glaciares y tendremos que construir muchas más represas artificiales con el fin de almacenar agua para los tiempos de estiaje (cuando no hay lluvias) y ser más eficientes en el uso del agua; la pérdida del recurso hídrico también traerá consigo la disfunción de nuestra capacidad para generar energía hidroeléctrica, que hasta hoy es la más barata, con lo que los precios de la energía podrían subir, afectando no solo a las poblaciones sino también a la industria, pues se vería disminuida su competitividad en mercados. En general, tendremos que generar nuevas fuentes de energía, de preferencia renovables y económicas para garantizar la seguridad energética en el ámbito nacional; la ventaja de hacer esto es que dependeremos menos de los combustibles fósiles, que además de ser costosos emiten gases de efecto invernadero.

- El nivel del mar podría elevarse entre 95 centímetros y 1,4 metros.Además de perder territorio nacional y tener que rehacer nuestros mapas, se incrementará el riesgo de las infraestructuras en la línea de costa: puertos, hoteles, carreteras, etcétera.

- Fenómenos climáticos exacerbados.- Lluvias más intensas, sequías más frecuentes. Según la CAF, el fenómeno El Niño de 1997-1998 causó a nuestro país pérdidas por cerca de 3.500 millones de dólares, equivalentes a 4,5\% del PBI nacional en esa misma fecha, y para tener una idea del retraso económico que este fenómeno causó, el megaproyecto Camisea aporta cada año el 1\% al PBI, necesitaremos 4 proyectos de la envergadura de Camisea para equiparar un impacto del fenómeno de El Niño; y según el Indeci, 


\section{Gráfico 3}

Retroceso de los glaciares en el Perú

\section{Evolución Pastoruri}
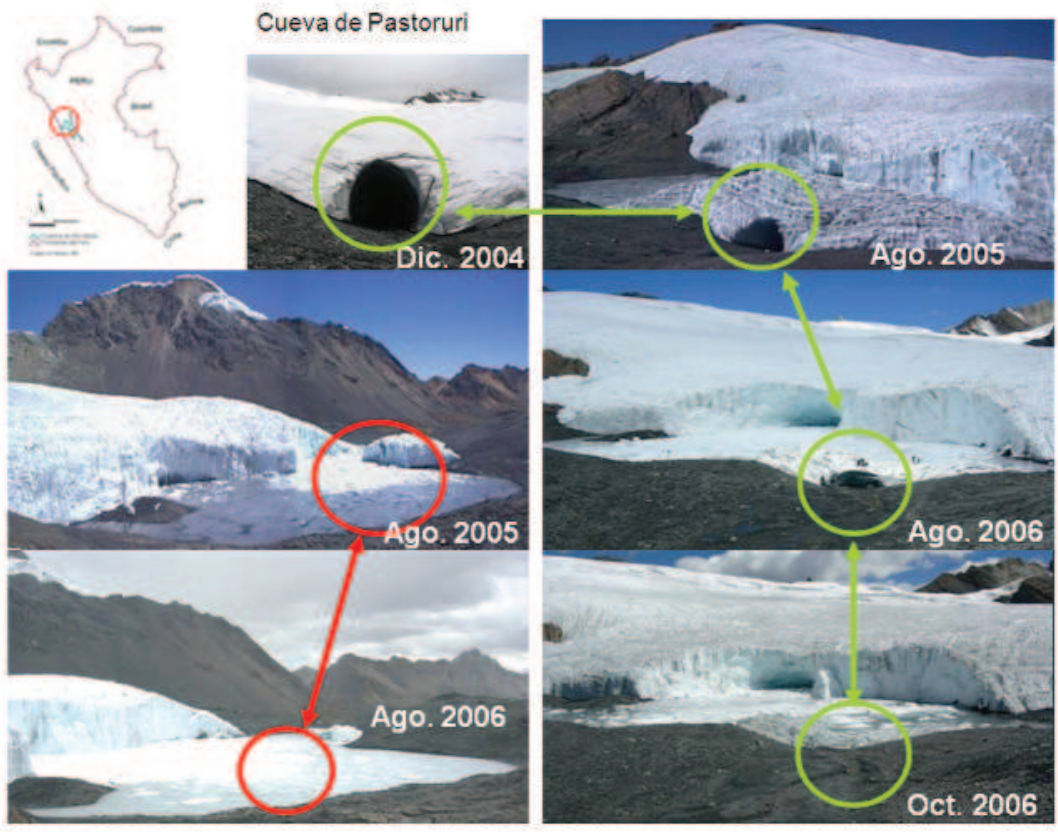

Retroceso del glaciar Broggi 1932-2004
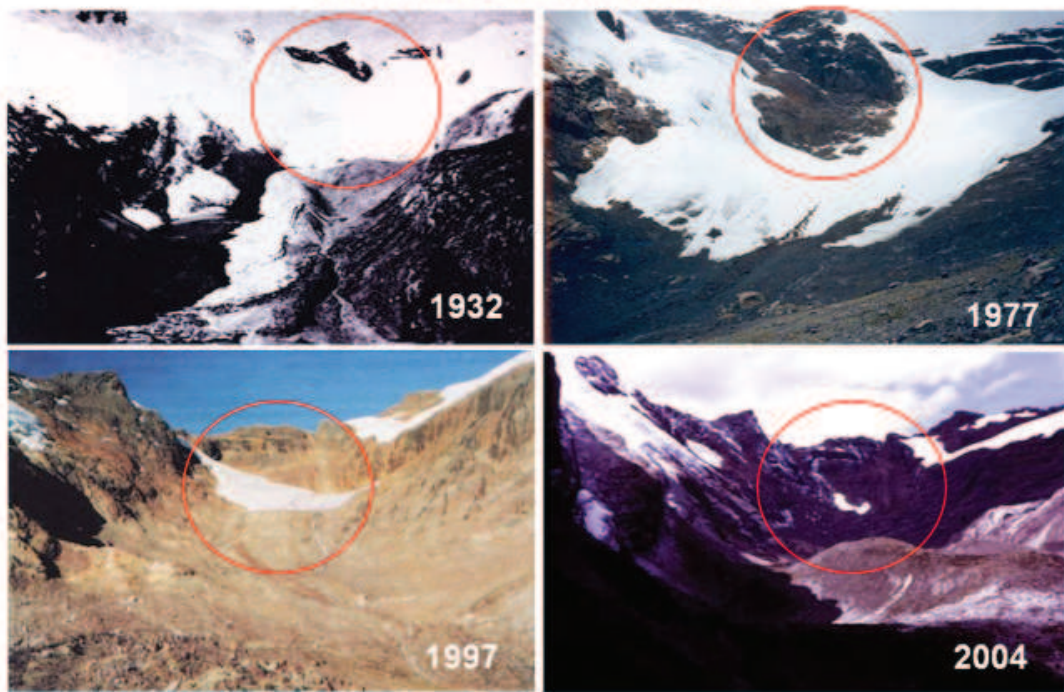

(continúa) 
(continuación)

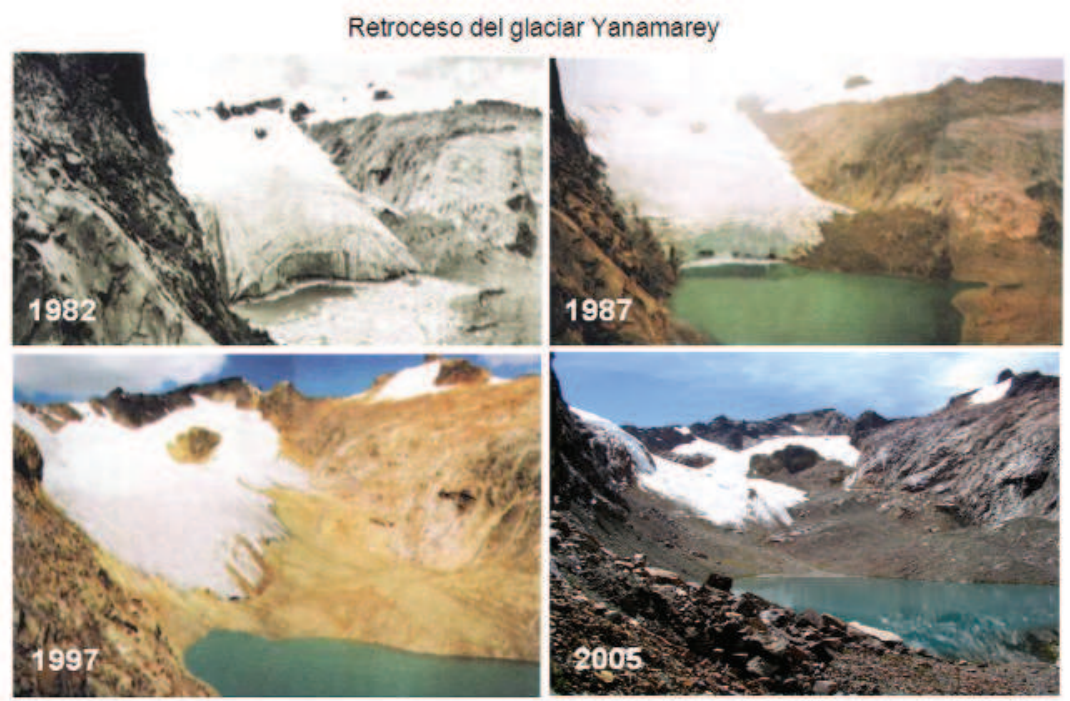

Fuente: Minag, Autoridad Nacional del Agua.

entre 1990 y el 2000 aumentaron más de 6 veces las emergencias por peligros naturales en todo el país y 7 de cada 10 emergencias fueron causadas por eventos climatológicos, que podrían hacerse más intensos y frecuentes por el cambio climático.

- Avance de la desertificación.- Con la consecuente pérdida de áreas agrícolas, recordemos que la zona de la costa del Perú recibe el $2 \%$ del agua disponible a nivel nacional, pero donde vive más del $70 \%$ de la población.

- Intensificación de vectores de enfermedades.- Además, aparecerán nuevas plagas y enfermedades o se enraizarán algunas como el dengue, la malaria y otras que afecten los cultivos y para las cuales aún no estamos preparados. Es probable que se tenga que incrementar el uso de pesticidas y medicamentos para controlarlos.

- Aumento de migraciones forzadas: en el caso del Perú, de las zonas rurales hacia las ciudades, en busca de mejores condiciones de vida. 
Entonces podríamos entender que el cambio climático nos va a afectar de manera directa, y si bien es cierto que afectará primero y con más fuerza a los más pobres, al no tener estos capacidad de respuesta, a largo plazo todos seremos perjudicados de una u otra manera.

Por eso, se recomienda revisar la Segunda Comunicación Nacional Sobre Cambio Climático, preparada por el Ministerio del Ambiente, que será enviada a la Convención Marco de las Naciones Unidas sobre Cambio Climático, en noviembre del 2010. Ahí se aprecia de manera detallada los impactos que nuestro país sufrirá, como consecuencia del cambio climático.

\section{NUESTRA CONTRIBUCIÓN}

Es cierto también que el Perú no ha contribuido mucho a este fenómeno, puesto que nuestro nivel de desarrollo no ha llegado a afectar de manera sustantiva el planeta; sin embargo, no debemos descuidar que los objetivos de desarrollo trazados pueden impactar y a la vez ser impactados por el cambio climático.

El Perú produce un $0,4 \%$ de los gases de efecto invernadero (GEI) del planeta (según estadísticas del año 2000), tanto como lo hace Nueva Zelanda o Dinamarca; sin embargo, los PBI de Nueva Zelanda y de Dinamarca, son 5 y 4 veces mayores que el del Perú, respectivamente. Si entendemos que las emisiones de GEI son generadas por el desarrollo, entonces ¿por qué el Perú no se desarrolla como estos países? La respuesta es evidente si analizamos las emisiones nacionales, donde el $47 \%$ provienen del cambio de uso del suelo (básicamente deforestación), que no genera ningún tipo de beneficio económico al país; es más, es considerada una actividad ilegal en el Perú.

Según el Ministerio del Ambiente, la superficie deforestada acumulada al año 2000 para la Amazonía peruana es de 7.172.554 ha, lo que representa el 9,25\% de la superficie de los bosques amazónicos y el 5,58\% del territorio nacional. El área total deforestada en la selva amazónica, durante el periodo 1990-2000, representa el 10,36\% de la superficie de los bosques amazónicos. La tasa anual de deforestación en el periodo 1990-2000, fue de 149.631,76 ha/año. La región San Martín es la que presenta la mayor superficie deforestada, con $1.327 .668,52$ ha $(18,51 \%)$. 


\section{Gráfico 4 \\ Inventario nacional de emisiones \\ Emisiones de GEI en el Perú, año 2000}

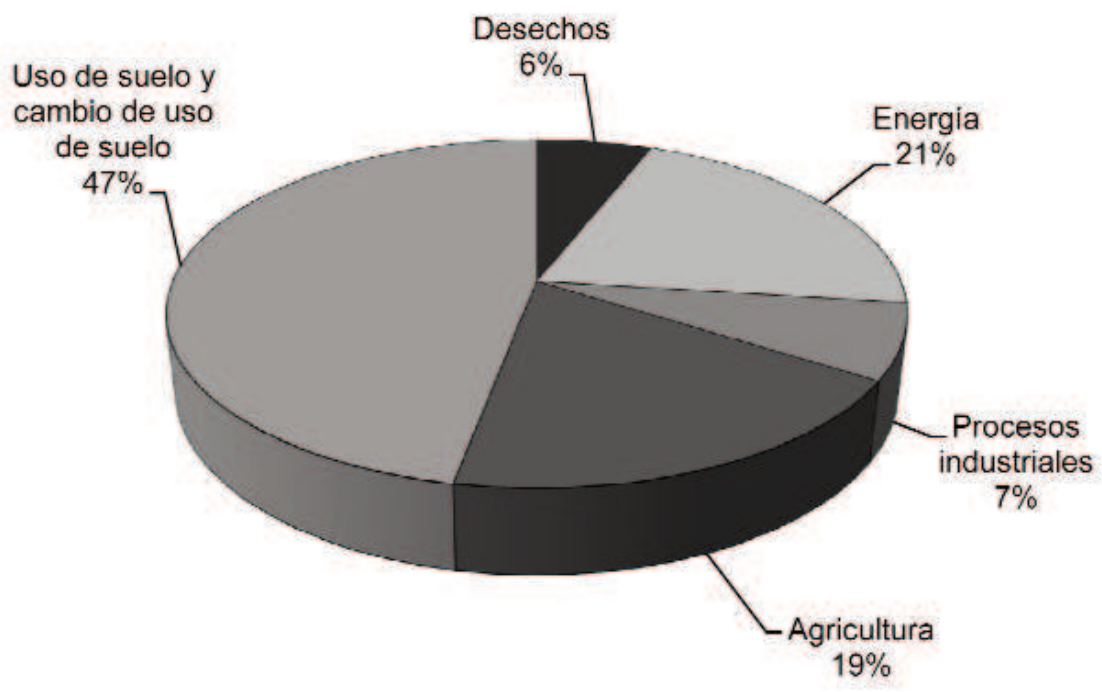

Fuente: Ministerio del Ambiente

Por dos razones, el Perú tiene las condiciones para apoyar las iniciativas globales con el fin de reducir las emisiones: la primera, para recuperar nuestra eficiencia, y la segunda, que mientras más disminuyan las emisiones a nivel mundial, las necesidades de adaptarnos serán menores en el futuro. La Secretaría de la Cmnucc ha estimado preliminarmente la cifra en inversiones y flujos de financiamiento adicionales necesarias en US\$210 mil millones por año para reducir en $25 \%$ las emisiones de carbono mundiales, mientras que las medidas de adaptación en el mundo costarán entre US $\$ 49$ mil millones y US $\$ 171$ mil millones anuales al 2030. Adicionalmente, el Ministerio del Ambiente ha calculado de manera también preliminar las necesidades financieras en mitigación y adaptación (como dos grandes rubros, sin especificar sectores o medidas) para el Perú, las que resultan entre US $\$ 431$ millones y US $\$ 453$ millones anuales al 2030 para mitigación, y para adaptación entre US $\$ 190$ millones y US $\$ 454$ millones anuales al 2030 (basados en cifras de la Cmnucc), o de US\$646 millones anuales al 2015, sobre la base del Reporte de Desarrollo Humano 20072008 , dinero con el que no contamos. Por este motivo, tanto la mitiga- 
ción como la adaptación al cambio climático a nivel nacional, se hace más complicada pero necesaria, ya que estas cifras deben verse como una inversión a largo plazo y no como un gasto.

\section{4. ¿QUÉ DEBEMOS HACER?}

Al mundo, y en especial al Perú, les urge tomar medidas drásticas para enfrentar el cambio climático y sacar provecho de este. Los costos y las situaciones de desastre y conflicto que se esperan por no iniciar las acciones hoy podrían ser inmanejables en el futuro, debido a que aun si se cumplen los esfuerzos de mitigación ofrecidos por los países desarrollados en los procesos de negociación internacional, el impacto económico será significativo. El PBI del Perú en el 2008 fue el equivalente a 65.000 millones de dólares (191.479 millones de soles) y según un estudio encargado por el Banco Central de Reserva (2009), bajo un escenario climático de un aumento de $2^{\circ} \mathrm{C}$ y $20 \%$ en la variabilidad de las precipitaciones al 2050, se establece que en el año 2030 alcanzaríamos un nivel de PBI total de entre 5,7 y 6,8\% menor que el nivel de PBI alcanzado sin cambio climático (PBI potencial); mientras que al año 2050 estas pérdidas respecto del PBI potencial serían superiores al 20\%. Estas medidas serían equivalentes a una pérdida promedio anual hasta el 2050 de entre $7,3 \%$ y $8,6 \%$ del nivel de PBI potencial. Sin embargo, si la implementación de políticas de mitigación globales surgiera efectos en estabilizar la variables climáticas al 2030, la pérdida promedio anual de aquí al 2050 se reduciría a casi la mitad, a un rango entre 3,9\% y 4,6\% del nivel potencial.

El Perú es un país altamente vulnerable y, por lo tanto, necesita una activa mitigación para evitar una carga creciente e inmanejable de adaptación. Se propone una mitigación global, ambiciosa y efectiva, liderada por los países desarrollados, pero donde participan también los países en desarrollo provistos de medios adecuados. El Perú no puede permitirse que la temperatura se eleve más de $1,8^{\circ} \mathrm{C}$, cifra indicada por el IPCC como el límite permitido que garantizaría un desarrollo adecuado de los pueblos.

Según la posición peruana llevada a las negociaciones mundiales de cambio climático, se considera que la mitigación debe ser liderada por los países desarrollados, los cuales no deberán poner ninguna condición a los países en desarrollo para la acción. Debería, además, estar 
muy claro qué parte de las reducciones se logra de manera doméstica en estos países, qué parte se logra con los mercados de carbono, y cuánto se logra por actividades relacionadas con la captura en bosques, así se podrá saber realmente cuáles serán las futuras concentraciones a nivel global y por ende las posibles consecuencias. Por lo tanto, sabremos claramente a qué nos enfrentamos para asegurar nuestro desarrollo y si debemos exigir mayores esfuerzos por parte de los países desarrollados.

Asimismo, y sabiendo que las metas son enormes, los países en desarrollo, como es el caso del Perú, deben mostrar una actitud proactiva respecto de la mitigación, que debe cubrir diversos aspectos, con escalas flexibles: desde medidas de educación (fundamentales en tanto evitan emisiones futuras), hasta metas voluntarias nacionales, pasando por proyectos, programas y políticas sectoriales. Además, se deben formular métodos efectivos para contabilizar las reducciones generadas por estas medidas.

Finalmente, y más importante aún, debemos iniciar la implementación de medidas de adaptación al cambio climático que nos permitan hacer frente a los efectos que ya son evidentes en nuestro país, garantizando nuestro desarrollo y sobre todo el de las comunidades más pobres y vulnerables frente al cambio climático.

\section{RECOMENDACIONES}

Luego de conocer el tema del cambio climático, de tomar conciencia de sus consecuencias y de interiorizar el problema, debemos preguntarnos qué podemos hacer nosotros como parte involucrada y afectada. A continuación se enumeran algunas recomendaciones básicas:

- Sembrar árboles y conservar las áreas verdes, que son sumideros de gases de efecto invernadero.

- Ser eficiente en el uso del agua y no desperdiciarla, ya que cada vez será más escasa y por ende más costosa.

- Ser eficiente en el uso de la energía, apagando y desenchufando los aparatos y luces que no usamos.

- Usar los medios de transporte más eficientes.

- No comprar productos que no necesitamos o no son esenciales para la vida, ya que se consume energía para producirlos. 
- Hacer más eficientes nuestros sistemas productivos cualquiera que sea la actividad a la que nos dediquemos, así ahorraremos dinero y ayudaremos al planeta.

- Usar combustibles más limpios, como el gas natural, el gas licuado de petróleo y los biocombustibles.

- Enseñar a los que no saben sobre las causas y consecuencias del cambio climático.

- Promover que los candidatos a elecciones locales y nacionales consideren en sus planes de gobierno los problemas del cambio climático.

- Participar en campañas de sensibilización.

\section{BIBLIOGRAFÍA}

Ministerio del Ambiente. "Posición nacional en el proceso de negociaciones de cambio climático" [en línea]. <http://www.minam.gob.pe>. [Consulta: 8 de julio del 2010.]

—. "Segunda comunicación nacional de cambio climático a la Convención Marco de las Naciones Unidas sobre Cambio Climático" [en línea]. <http://www.minam.gob.pe>. [Consulta: 8 de julio del 2010.]

Panel Intergubernamental sobre Cambio Climático-IPCC. "Cuarto reporte de investigación del Panel Intergubernamental sobre Cambio Climático" [en línea]. <http://www.ipcc.int>. [Consulta: 8 de julio del 2010.]

Senamhi (2010). Escenarios nacionales de cambio climático. Lima: Servicio Nacional de Meteorología e Hidrología del Perú. 